\title{
A study on medical Internet of Things and Big Data in personalized healthcare system
}

\author{
V. Jagadeeswari ${ }^{1}$, V. Subramaniyaswamy ${ }^{1 *}$, R. Logesh ${ }^{1}$ and V. Vijayakumar ${ }^{2}$
}

\begin{abstract}
Personalized healthcare systems deliver e-health services to fulfill the medical and assistive needs of the aging population. Internet of Things (IOT) is a significant advancement in the Big Data era, which supports many real-time engineering applications through enhanced services. Analytics over data streams from loT has become a source of user data for the healthcare systems to discover new information, predict early detection, and makes decision over the critical situation for the improvement of the quality of life. In this paper, we have made a detailed study on the recent emerging technologies in the personalized healthcare systems with the focus towards cloud computing, fog computing, Big Data analytics, loT and mobile based applications. We have analyzed the challenges in designing a better healthcare system to make early detection and diagnosis of diseases and discussed the possible solutions while providing e-health services in secure manner. This paper poses a light on the rapidly growing needs of the better healthcare systems in real-time and provides possible future work guidelines.
\end{abstract}

Keywords: Healthcare system, Internet of Things, Sensors, Security, Big Data analytics, Mobile computing, Cloud computing, Fog computing

\section{Introduction}

Healthcare sector is one of the largest sectors in the developing world with the annual increase in revenue and employment [1]. In early days, diagnosis of rare diseases can only be detected by a complete physical and analytical analysis made within the hospital premises. Nowadays, a smartwatch can help us to diagnose any irregularity with our Health. For example, the irregular heartbeat of an elder people. In case of an epidemic disease such as Ebola, technology plays a major role in controlling the rapid spread of this incurable disease by informing the people to take appropriate steps. The patients should not consume medicine without the presence of doctors advised by disease control and prevention (CDC) [2]. In contrast, recent technological advancement brings the preliminary diagnostics to the patient's doorstep. Due to advancement in technology, healthcare services are shifting from hospital-centric care to personalized individual-centric services $[3,4]$. For

\footnotetext{
*Correspondence: vsubramaniyaswamy@gmail.com

1 School of Computing, SASTRA Deemed University, Thanjavur, India Full list of author information is available at the end of the article
}

example, several clinical procedures such as blood testing, diabetic monitoring, pressure monitoring can be done at a remote location in a real-time manner. Due to the advancement in telecommunication and data services like Data as a Service (DaaS) in the developing countries provides remote monitoring healthcare system feasible and serviceable. With the rapid development of the new telecommunication services, wearable IoT sensors, cloud computing, fog computing, and mobile computing provide real-time monitoring of users, diagnosis, communication with doctors and prescribe medicines and deliver at doorsteps are applicable in the better way.

Internet of Things (IoT) expands the independence of humans to interact, contribute and collaborate on things. IoT gradually developed heterogeneous technologies with sophisticated protocols and algorithms. It plays important in global communication among millions of devices with wired/wireless sensors, electronic appliances like Television, Refrigerator, etc., connected to the Internet [5]. Then it plays an amazing role in many fields such as Agriculture [6, 7], healthcare [8, 9], entertainments [10], automobiles [11], sports [12], home [13, 14], industrial appliances [15], business [16], etc. The reasons 
for the rapid development of the IoT are high accuracy, low cost, and reduce time by predicting the future in a better way. The IoT gadget like sensors and actuators were embedded into physical devices such as home appliances, vehicles, and smart industrial machines to monitor and exchange data using wireless networks with the help of Bluetooth, Zigbee, IEEE 802.11 (WI-FI), WiMAX, etc. The sensors are implantable in the body or wearable that senses the physiological states like blood pressure, heart rate, body temperature, pressure rate, electrocardiogram (ECG), electroencephalogram (EEG) [17]. Monitoring ailing people are more comfortable for people who had elders, children alone in the home. The sensor connected to the users, frequently monitor the user $24 / 7$ and provides alerts on user's changing health condition to the family, doctors, and emergency services. The growing needs of better technologies for addressing real-time issues in processing larger data without compromising security and privacy requires significant attention from the global research community.

Big Data analytics [18-21] emerged as the good solution for the process of abundant data and improves decision-making characterized by 6 V's: volume, variety, velocity, veracity, validity, and volatility. The main characteristic is volume, that refers to a quantity of information being used and analyzed to obtain the desired result. It raises a flood of data from various sources includes sensors, scanners, mobile phones, digitizers, video, email, social networks, and the internet. Variety refers to the type of data stored, analyzed and processed. The type of data may be text, images, videos, sound, geometrics, and the combination of each type. Veracity refers to the accuracy of data is good enough for Big Data. Big Data also presents a challenge to store, process and assist the data effectively [22]. To overcome the problems of Big Data, cloud computing $[23,24]$ emerges as a fundamental support to store and process the resources data, accessed from any location through the internet. Accessing resources stored in the cloud categorized into the public cloud, private cloud, and hybrid cloud. The resources of the public cloud can be accessed, shared and processed by third-parties. A private cloud allows the resources accessed by the organization. A hybrid cloud combines both public and private clouds, often used to supplement internal data with public orchestration. Software as a Service (SaaS), Infrastructure as a Service (IaaS), Platform as a Service (PaaS) is the cloud services or resources available to users on demand via the internet. As the data increases day-by-day, the vast amount of data have to be collected, analyzed, managed, and stored, in the cloud which increases the complexity of cloud-based services. To address this issue, we introduce fog computing [25-28] at the edge of the cloud for pre-processing of generated data. Fog improve the efficiency by reducing the data transfer to the cloud transform from physical devices. Fog enables a secure data transaction within millions of devices connected, ensures high scalability and even distribution of data. Big Data analytics is a voluminous data mining approach which requires both cloud globalization and fog localization.

Among many self-tracking devices, a smartphone $[29,30]$ is a user-friendly device that provides real-time remote healthcare system connected with users and clinical staff. Globally, development and the utilization of the smartphones have enhanced the performance of healthcare applications by providing efficient services to users. It monitors both active data and daily behavioral activities from embedded mobile sensors [31]. It has a pocketsized display with wireless network communication and battery power to process the hardware-software system. Smartphone health monitoring application involves a remote healthcare system improved with the advancement of mobile, communication networks, sensing, and embedded devices. The mobile-based application development has paved the possible way for directly accessing and analyzing the patient's information from the sensors connected with users. Mobile healthcare applications provide faster delivery of information with reduced cost, unlimited Internet-based services, and have efficient battery backup.

The main objectives of this survey are multifarious:

- Examine the role of cloud computing depicts the healthcare system with general architecture.

- Illustrating a high-level comparison between cloud and fog computing and providing secure privacy of patients with fog computing.

- Augmentation of the IoT healthcare application with IoT-based healthcare monitoring architecture.

- A focus on healthcare monitoring system in Big Data era.

- Interactive healthcare applications are reviewed with a smartphone.

- Providing the pros and cons of the healthcare system took for the survey.

- Exploring current challenges in the personalized healthcare system.

- Discussion on a promising solution for the real-time healthcare monitoring problems.

The remainder of this article is organized as follow. "Role of cloud computing in healthcare systems" section review the role of cloud computing in the healthcare system. "Securing privacy of patients with fog computing" section describes the security privacy of patients with fog computing. "Enhancement of healthcare applications 
using Internet of Things" section review the healthcare application using the IoT. "Development of healthcare sector in the Big Data era" section evaluates the healthcare sector in the Big Data era. "Interactive healthcare applications with smartphones" section assess an interactive healthcare application using smartphones. "Current challenges in the personalized healthcare" and "Discussions on the promising solutions for the real-time problems" sections discuss the current challenges and real-time solutions for the personalized healthcare system. Finally, "Conclusions" section provides our concluding remarks.

\section{Role of cloud computing in healthcare systems}

In the real world, the lack of healthcare and support system to the senior population is still an existing challenge. To overcome the challenge, Pham et al. [32] delivered a real-time smart home healthcare service for elders staying alone. The healthcare service adopts Software-asa-Service (SaaS) cloud architecture with four layers: the service presentation layer, cloud engine layer, the data processing layer, and the cloud storage layer. Non-invasive sensors used to collect physiological, motion and audio signals of the users. Contextual information [33, 34] is collected such as the user's activities and location details through the optitrack camera system. MYSQL is a relational database to store structured data like user information and NoSQL is used to store sensor data.
Based on these collected data, the users are monitored continuously. For instance, a user's physiological signals are generally considered as contextual information for prediction and analysis of the state. In a scenario of vigorous running a fast heart rhythm is expected from the user whereas, an abnormal heart rhythm from the user in an immobile state triggers the alarm. The authors also developed a robot assistant which track the dehydration level of the user. In this system, the water content level of the user is frequently checked by the robot to instruct the user to drink water during dehydration. Gradient boosting decision tree algorithm is used in robots to recognize the body activities. Robot assistant is a case study implementation of the cloud-based smart home environment (CoSHE). Non-invasive sensors are used to monitor the entire home, so the energy consumption is too high.

The role of cloud computing in healthcare is described in Fig. 1. The IoT devices and sensors acquired user health data information and relayed to the cloud with the help of a wireless communication network. The monitored information is stored in the cloud repository, and the data are preprocessed to extract, analyze, and to determine a diagnosis based on the knowledge base in which medical books, references, and advice are predefined for diagnosis. Based on the user health state, the alert message is generated to doctors, ambulance, and caregivers. The system helps to diagnosis at the initial stage, so we provide better health care by taking early

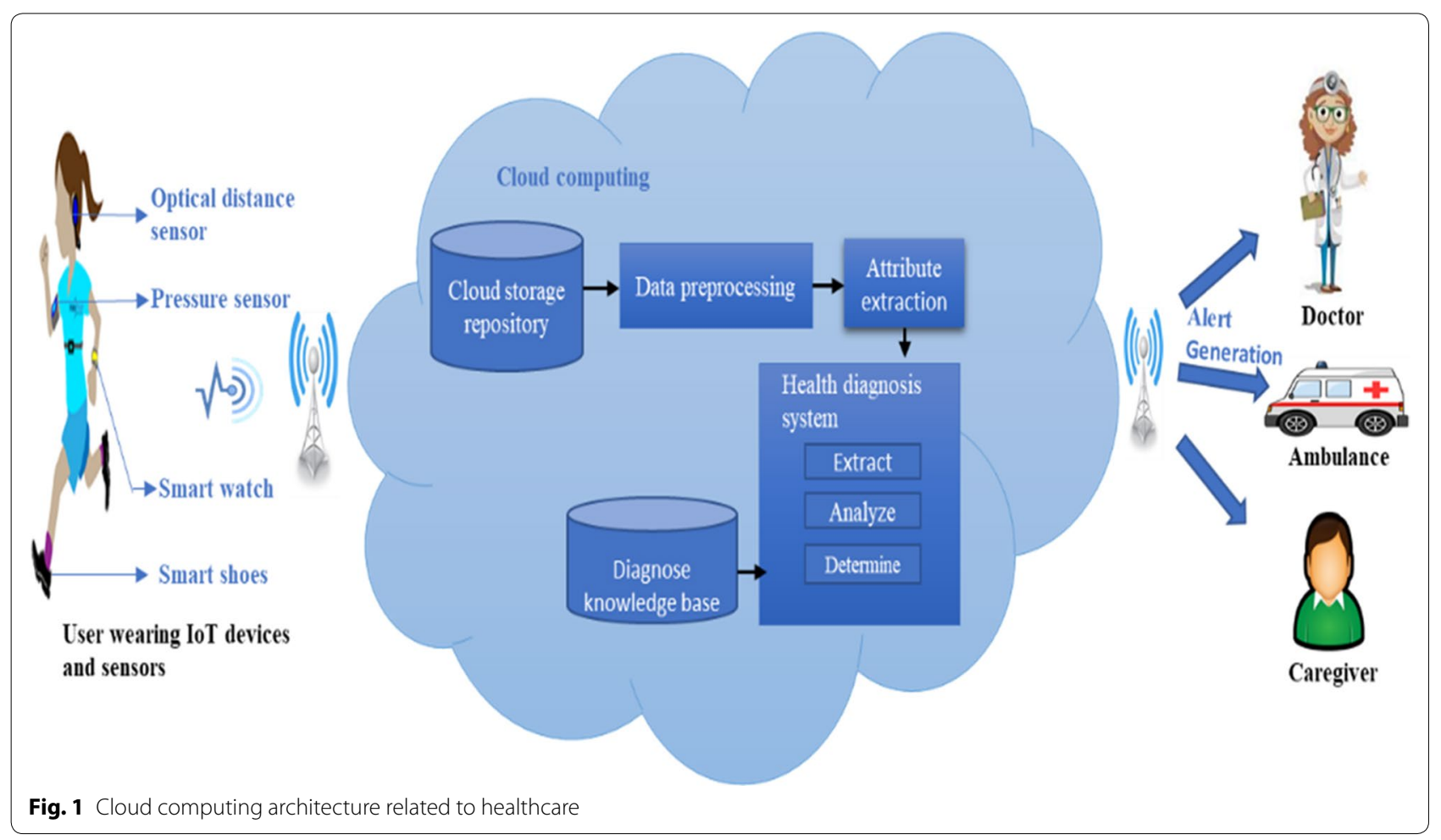


precautions. Table 1 describes the methodology used, approaches, sensors, advantage and disadvantage of the cloud-based healthcare system.

To predict the feasible disease in the users, Verma and Sood [35] designed a framework to generate an alert message. Using condition-based methodology, the cloudcentric diagnostic system is proposed to forecast the possible diseases in the user. Medical devices and sensors are used to monitor the frequent changes in health parameters of the users based on time interval. The monitored information collected and passed to the local processing unit, it may be a smartphone or portable devices. The data are analyzed in the cloud and generate a User Diagnosis Result (UDR) which consist of user's profile information, possible disease, severity, and the probability of occurrence of diseases. The probability is calculated by diagnoses of user-sensor data, context-specific information, and doctor's report. The security is provided by the Trusted Third Party (TTP) entity by encrypting user password with the private key. Finally, UDR result is generated by three conditions: scale-based, pattern-based and frequency-based conditions. Examine the probability occurrence of diseases, the user divided into the safe and unsafe state. The alert generation algorithm tests the user's state, and the messages are sent to doctors, caregivers and ambulance in need of emergency assistance. The authors conduct experiments by deploying the smart student diagnosis system where students are monitored through sensors. The results are analyzed based on comparing it with a decision tree, k-nearest neighbor, naïve Bayes, support vector machine. Decision tree acquires superior performance compared to other algorithms.

The cloud-based system designed a framework [36] to prevent and control the Zika virus. In this system, the user registers their personal information in the mobile application, and a unique id is generated for each user. Users enter into the application using their respective unique id and enter ' $\mathrm{Y}$ ' or ' $\mathrm{N}$ ' for the symptoms in the form of attributes. Based on the user symptoms-response for the health-related attributes in mobile phones, the user is classified into infected or uninfected using a Naïve Bayesian Network algorithm. The wireless mosquito sensors are used to track the mosquito dense site location, dense mosquito site, breeding site location, temperature, and humidity. The tracked information used to plot in Google map using Geographic Positioning system (GPS) about mosquito dense and infected user location areas. Using Google map, the uninfected users' can re-route their path to safeguard them. The risky location information is sent to the government, healthcare department to take adequate measures and prevent the spreading of the virus. The system yields high precision regarding identifying the risky location with the help of the Global Positioning System. The system is estimated by 2 million users dataset in Amazon EC2 cloud afford high accuracy in the diagnosis of Zika virus and GPS risk assessment.

H1N1 is an air-borne disease spread easily, affect large population and need superfluous amount to diagnose [39]. Sandhu et al. [37] reviewed the widespread of the H1N1 virus and proposed a cloud-based system to monitor and control the virus. With the help of mobile phones or web browser, the user's response data is collected and sent to the cloud where the decision making is effective. The symptoms are classified into primary, secondary, tertiary and high-risk conditions. Based on user's symptoms-response, infected and uninfected peoples are classified. Infected users are continuously monitored until they get rid of the H1N1 disease. Information and suggestion box of the system component is used to collect all information about users, doctors, hospitals and send up-to-date information and suggestion about the H1N1 disease to the user via SMS or e-mails. The doctors are allowed to upload only the patient's details, treatment process not personal information of the patients.' An Outbreak Role Index (ORI) is used to determine the probability of the user to spread or receive the virus. Social network analysis generates the graph for the infected user, and it is useful to the uninfected users about regional exposure. The system implemented in AmazonEC2 and performance of classification results with high accuracy.

The IoT devices, sensors, and cloud computing jointly present a real-time healthcare infrastructure to widely increasing disabled and older adults. Hossain and Muhammed [38] designed a framework for continuously monitoring of disabled and/or elder people through sensors. The advancement of cloud computing creates a platform to gather a tremendous amount of information tracked by the sensors and accessed by anyone at any time everywhere. The IoT devices and sensors used to track electrocardiogram (ECG) continuously, and the information needs privacy, so ECG signals are enhanced before processing. The gathered patient's information is stored in MYSQL before moving to the cloud and accessed by healthcare units. Watermarking and signal enhancement are used to secure the data on the client side. The information is watermarked on the client side and sent to the cloud for feature extraction and classification. Again reconstruction, watermark extraction in client side. So the specific user information is shared only with desired healthcare services, and quality is improved. However, the system accuracy is untested. 


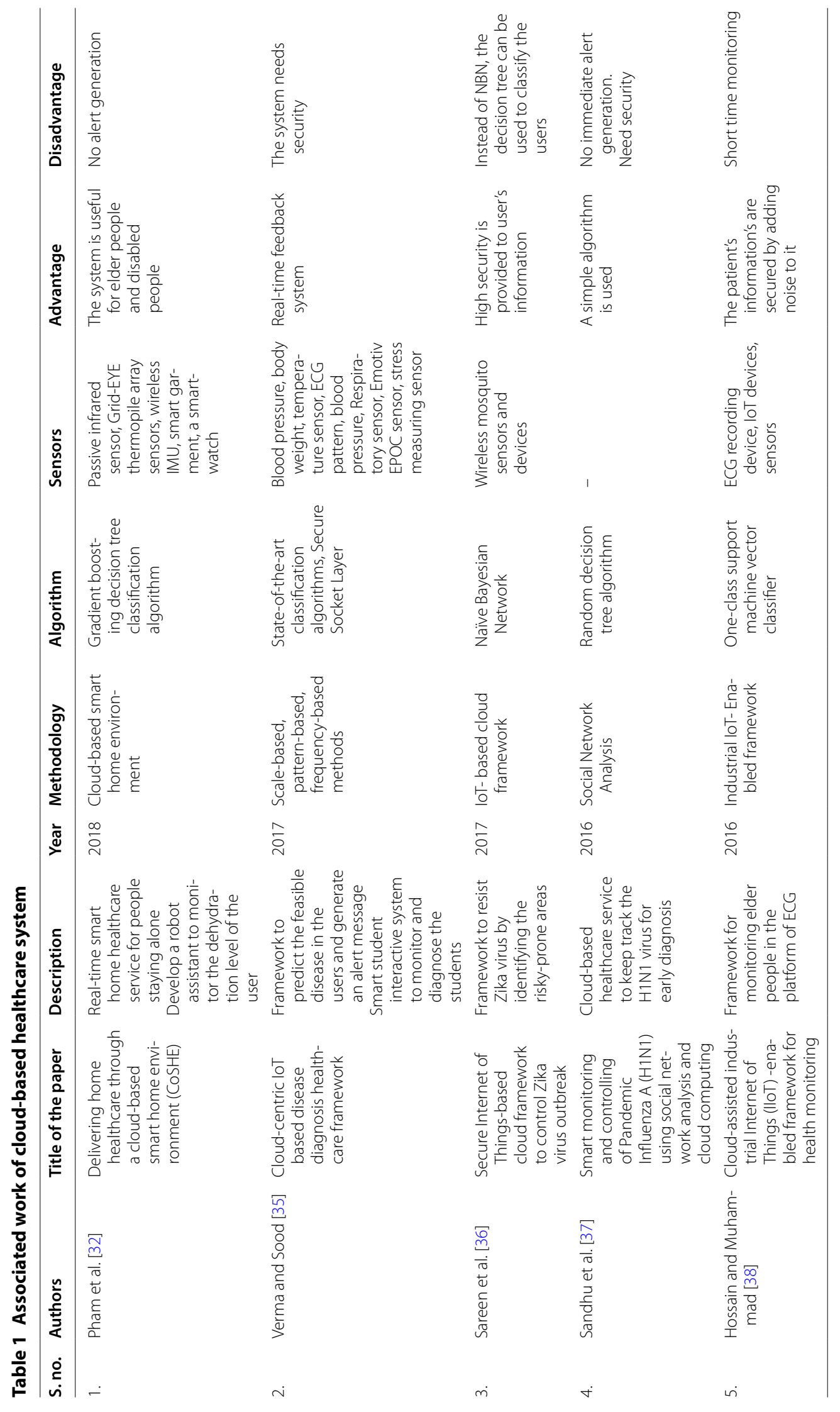




\section{Securing privacy of patients with fog computing}

IoT generates a vast amount of data to be processed in real-time and delay is caused due to the transfer of data between cloud computing and end-user. To diminish the delay, Verma and Sood [40] proposed a fog assisted remote monitoring healthcare services and real-time alert generation. The system architecture consists of three layers: device layer where the IoT devices and medical sensors collect information about the user, fog layer analyze the patient's information using classification rules, and cloud layer notifies warning alerts to the family members. The intermediate fog layer is more suitable for real-time analysis of sensors data with low latency and high quality of service. Two types of communication are engaged between fog and cloud layer: conventional communication updates the cloud with patients information and smart communication retrieve the queried patients history information from the cloud. The patients address their health-related information on their mobile phones, and the unique patients identification code is generated for each patient. Then event classification classifies the patients into normal and abnormal through Bayesian Belief Network (BBN) based on patients information. The Degree of Impact (DOI) is the probability of event calculated based on health, environment, and behavior related attributes of the patients. When DOI exceed the level, then fog layer sent a signal to cloud for further analysis. In the cloud, again the patients classified into the safe and unsafe state. The temporal health index is calculated for unsafe state patients, and the real-time alert message is generated to responder and hospitals. The proposed system validated by 67 patients monitored at smart homes in the AmazonEC2 cloud and yields a better result. Effective decision making and real-time alert generation are achieved in fog assisted IoT enabled system.

In the developing countries, the diagnostic test for chikungunya is not easily accessible and reasonable. Sood and Mahajan [41] designed a framework for fog assisted healthcare system in diagnosing and preventing the chikungunya virus. Early diagnosis of chikungunya is too hard for healthcare services. To solve this, the authors proposed an effective early diagnosis at home or remote areas to minimize the mortality rate. The users are continuously monitored and the data are collected in the cloud. The authors designed a fog assisted cloud-based healthcare system in which fog is introduced between the mobile and cloud to analyze the real-time data at the edge of the network. The data are processed in fog and finally stored in the cloud. The author classifies the user's into five different categories: infected, uninfected, exposed, susceptible, and exposed based on the symptoms track by wearable sensors embedded in the user. The user's infection is categorized based on their symptoms using a
J48 decision tree. Compared to other classification algorithm $\mathrm{J} 48$ results with high accuracy and reduce response time. Temporal network analysis is used to differentiate the risky infected location, safeguard the uninfected user, stay away from the infected user and generate an alert message to the government agencies in the direction of taking immediate control steps. For diagnosing chikungunya, the system provides better performance and active distribution of messages in the healthcare system.

Fog is also known as edge computing, which is the extension of cloud computing created by Cisco. The execution part of fog computing in healthcare monitoring system is depicted in Fig. 2. The fog is an intermediatory between the end user and cloud to reduce the latency and improve the quality of service. Fog analyze the real-time sensitive data at the edge computing instead of sending a huge amount of data to the cloud. IoT devices like smartwatch and smart dress track user information and transmitting sensing information continuously to fog using wireless protocols such as Bluetooth, Wi-Fi, etc. Fog layer generally communicates with several protocols and have multiple interfaces to provide effective response [42]. The data are analyzed, notified, processed, classified into infected and uninfected users using an algorithm, and the real-time decision is made in fog, and finally, data are stored in the cloud. From the cloud, the message generated to doctors, hospitals and family members. The Table 2 illustrates the pros and cons of the fog-based healthcare system.

Sareen et al. [43] proposed a secure system to predict and prevent the Zika virus with the integration of IoT sensors, cloud computing, fog computing and mobile phones. The system reduces the communication cost and latency time by switched the fog computing in between the cloud and end user. Mobile phone and sensors are front-end section connected to back-end cloud via middle section fog computing. The user registers their name and personal information in the mobile-based application. For each registered user, the unique reference number is automatically generated. The users communicate with doctors, healthcare agencies, public authorities, hospitals by providing their reference number. The fuzzy $\mathrm{K}$-nearest neighbor algorithm is used to classify the users into infected and uninfected based on their symptoms. Preventive measures are provided to the users who are diagnosed as uninfected. The user's locations are automatically tracked through Geographic positioning system in mobile phones. Along with that, mosquito sensors discover the breeding sites in risky areas and the sensed information are used to plot the infected users in Google map and message sent to uninfected users about the risky location to safeguard them. The details of risky location are sent to the government to take preventive and control 


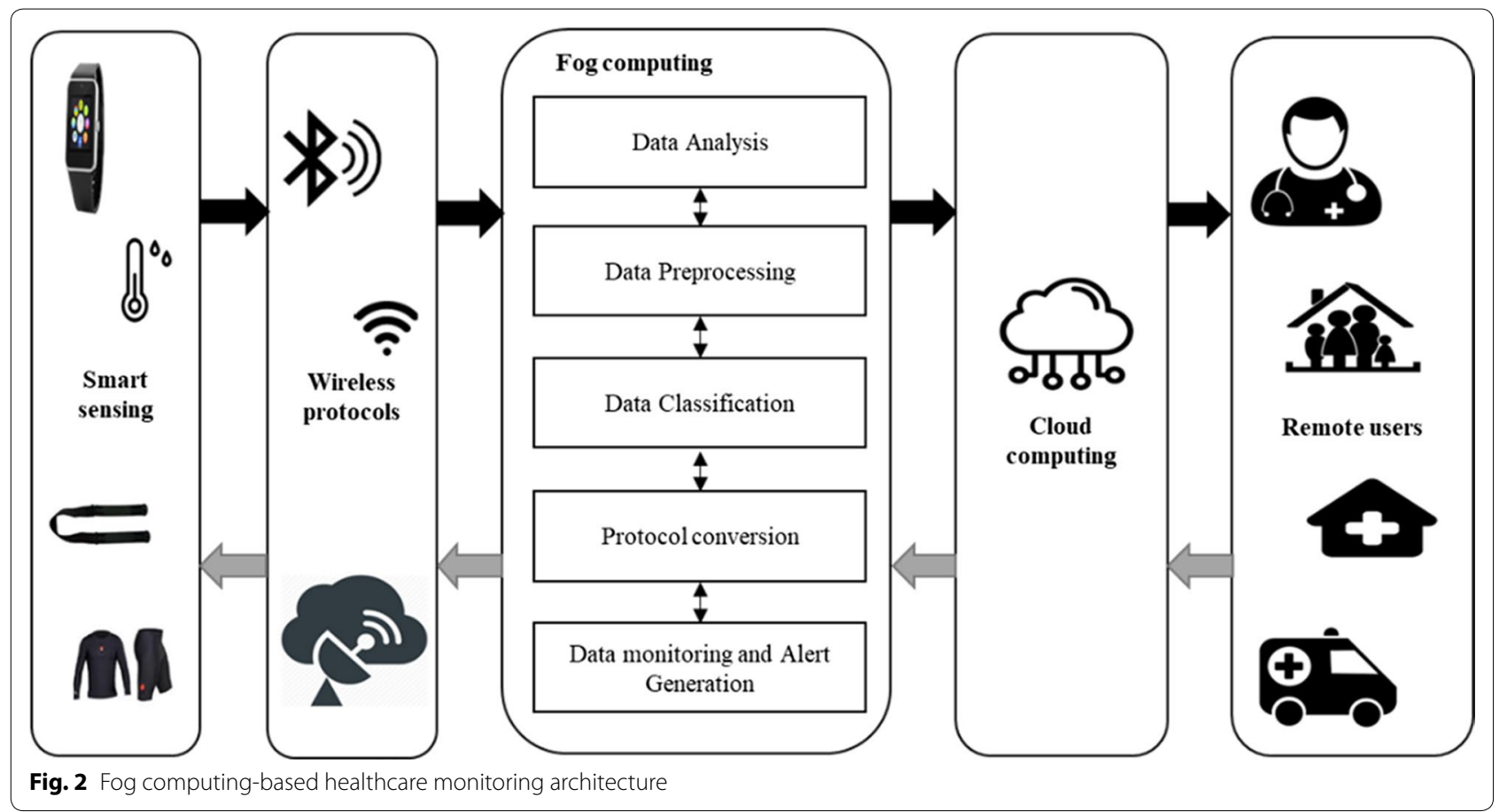

measures. The system is implemented in Amazon EC2 cloud and provide the efficient result of high accuracy for 94.5\% of million users in the early diagnosis of Zika virus outbreak.

Sensors or actuators are connected to many physical devices to gather a huge amount of information. Not only in the medical field, sensors also play a vital role in transportation, security, business, and manufacturing. Traditional cloud computing is a centralized distribution, so the system moves from cloud to fog computing to conserve network bandwidth, address a security concern, operating reliability, access more data and process it efficiently. In the cloud, the data are collected, stored, analyzed, processed to make a decision and notified wherein fog; the data are analyzed, notified, processed and finally stored in the cloud. For real-time interactive services, fog provides better performance compared to the cloud. Nandyala and Kim [44] proposed a C2F architecture for U-healthcare monitoring for smart homes and hospitals. Through sensors, users are monitored in homes and hospitals. The data are analyzed, and the message is sent to doctors indeed for an emergency. However, the real-time smart homes and hospitals system yet to be deployed and its accuracy remains untested.

The feature of electrocardiogram (ECG) $[46,47]$ is extracted as a study of healthcare IoT. Many cardiac diseases are diagnosed using ECG, and the patients should be monitored 24/7 continuously. The huge amount of data have been transmitted over the network, so the possibility of occurring error also high. The single error like bit error, packet dropping, and transmission latency lead to increase mortality. To overcome the issue; Gia et al. [45] proposed the fog layer between the gateway and remote cloud server to improve the quality of service, reduce the transmitted data and save network bandwidth. The ECG feature extraction template contains ECG preprocessing, wavelet transformation, and ECG feature extraction. They have exploited a light-weight transform mechanism to extract heart rate, $P$ wave, and $R$ wave. The extracted patients information is sent to the doctors to take immediate treatment in case of an emergency. Geographical location of the user is determined by tracking device, and immediate actions are taken by caretakers. However, electrooculography [48], electromyography [49], electroencephalography [50], electrocardiogram are important to diagnose human abnormalities, yet ECG extraction only proposed. Currently, real-time remote monitoring of EEG to record the electrical activity of the brain is proposed yet not implemented.

\section{Enhancement of healthcare applications using Internet of Things}

The students while working on radio frequency identification (RFID) at MIT in late 2000, announced the word "IoT" [51, 52]. Healthcare monitoring architecture using IoT depicts in Fig. 3. IoT devices integrated with the sensors like wearable IoT sensors, climatic sensors, temperature sensors, etc., to track users physiological 


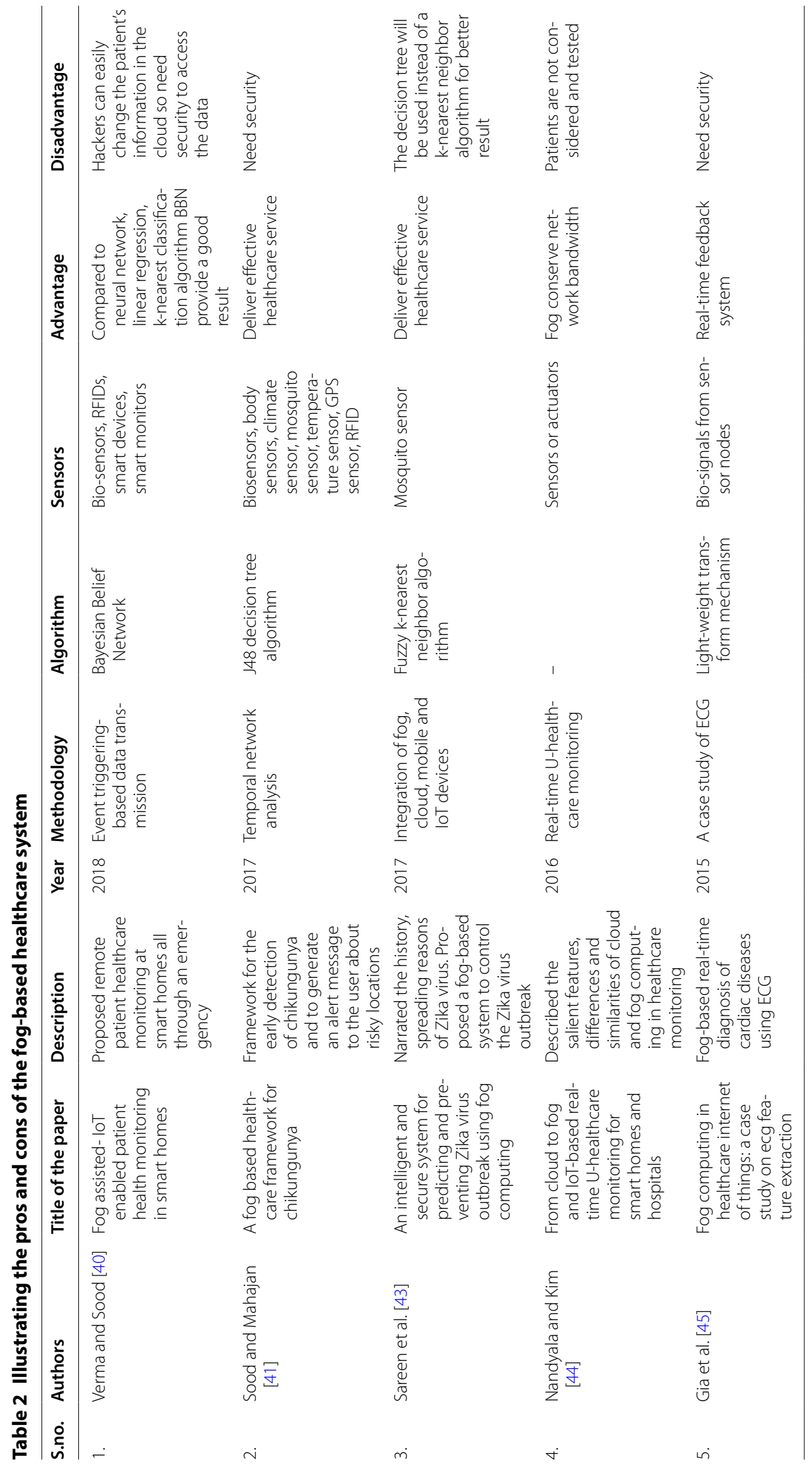




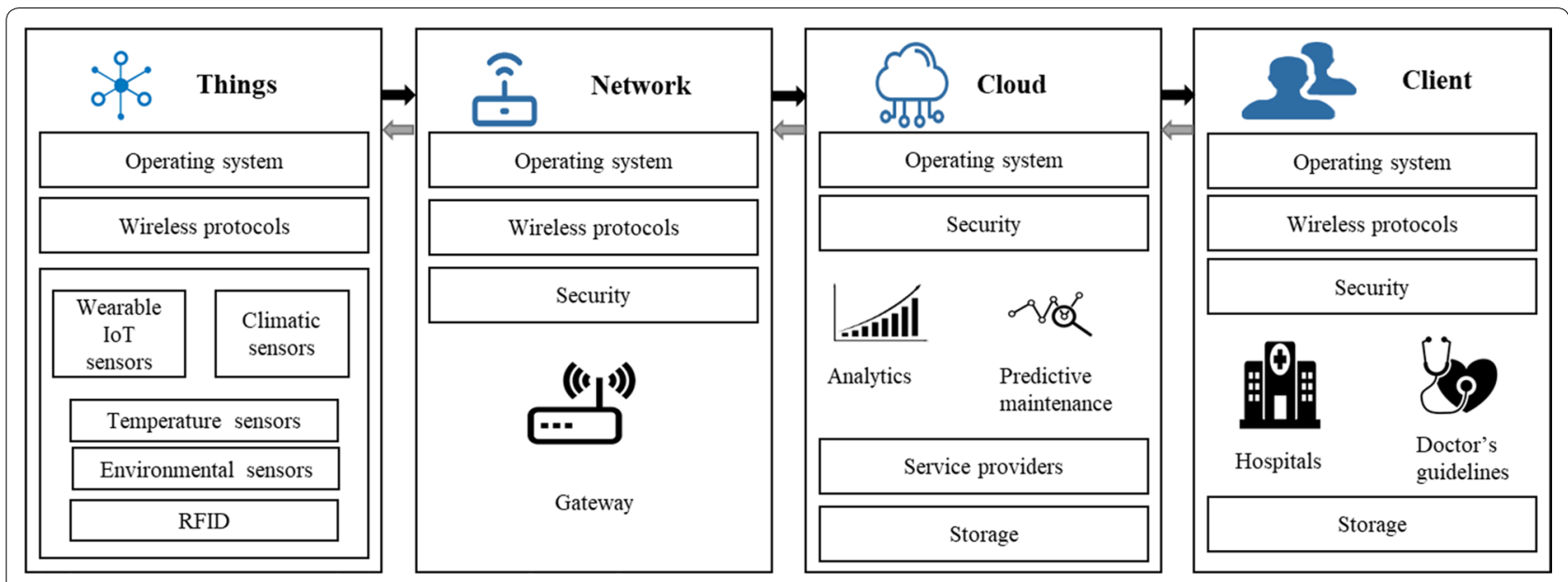

Fig. 3 loT-based healthcare monitoring architecture

information, atmospheric information, and temperature. Physiological information such as body temperature, diabetes, blood pressure, heart rate monitored by the body sensor and biosensor. For instance, smartwatch determines the body temperature, oximeter, accelerometer, and track GPS location. Sensors embedded in clothing track ECG, EMG, pressure rate of the patients. The observed data transferred to the cloud through networks for storage and message is conveyed to clients, i.e., doctor's mobile phone/PDA, family members, and hospitals. Table 3 mentioned below represent the methodology, algorithm, advantage, and disadvantage of the IoT enabled healthcare system.

Traditionally ICU patients are monitored by the nurse 24/7 alternatively. Still, it is a challenging task because Small mistake or error also lead to patients' death. Prajapati et al. [53] proposed a real-time IoT-based system to monitor the ICU patients continuously. Bedside patient monitor is used to screen the invasive and noninvasive blood pressure, temperature, capnography, ECG, FASTspO2, respiration, conventional diagnostic, BIS and cardiac output. Bedside patient monitor continuously transmits the heart rate, oxygen level, blood pressure, pressure in the brain of the patients through interconnection networks to the server. The probe can be inserted into the brain to measure the intracranial pressure. Here, mobile phones or personal digital assistant (PDA) is used to communicate with doctors. The real-time system analyzes the patient's data, track the parameters until crosses its boundary immediately notification is sent to the Emergency Care Unit and appropriate doctors. The system provides patients' information to the concerned doctors to take precautionary steps. The authors designed architecture to monitor the ICU patient, minimize the possibility of human errors and improve fast communication.
The rapid increase of diseases, insufficient health advisors, need for continuous monitoring of discharged, elder people lead to the necessity for Technology-Enabled Care (TEC). Baali et al. [54] designed power-aware wearable devices connected with the IoT for the real-time healthcare system and revised the state of signal processing, power management, communication, and computing architecture for IoT sensors. Wearable sensors play a vital role in TEC to measure and monitor ECG, blood pressure, heart rate, the oxygen level of patients without affecting their regular activities. The sensors directly connected to web-based services to transfer messages through wi-fi or 4G network. Biopotential signal processing includes ECG, EEG, cardiovascular diseases (CVD), Electroencephalography require a Holter monitor to screen 24/7 patients continuously with the help of different types of electrodes. Through electrodes in sensor provide low power consumption and excellent user-friendly wearable devices. Biopotential electrodes skin-interface categorized into dry, wet, insulated and non-contact electrodes altering ionic current in the body into the electronic current. Blood pressure (BP) sensor used to monitor the hypertension of the patients by wearing an inflatable bag around the arm. Ambulatory $\mathrm{BP}$ is frequently monitored lead bruise under the cuff. Oxygen level measurement calculates the cardiorespiratory health grade based on blood oxygenation in real-time. Inertial sensors are used to track the human physical activities. In real-time sensors monitor, collect the information, analyzed, processed (filtering, segmentation, compressed) and features are extracted before transmitting to the cloud or any database.

The concept of IoT is emerging in smart transportation, smart education, smart cities, smart business and smart manufacturing. Smart health bridge the gap between electronic health and electronic-governance in 


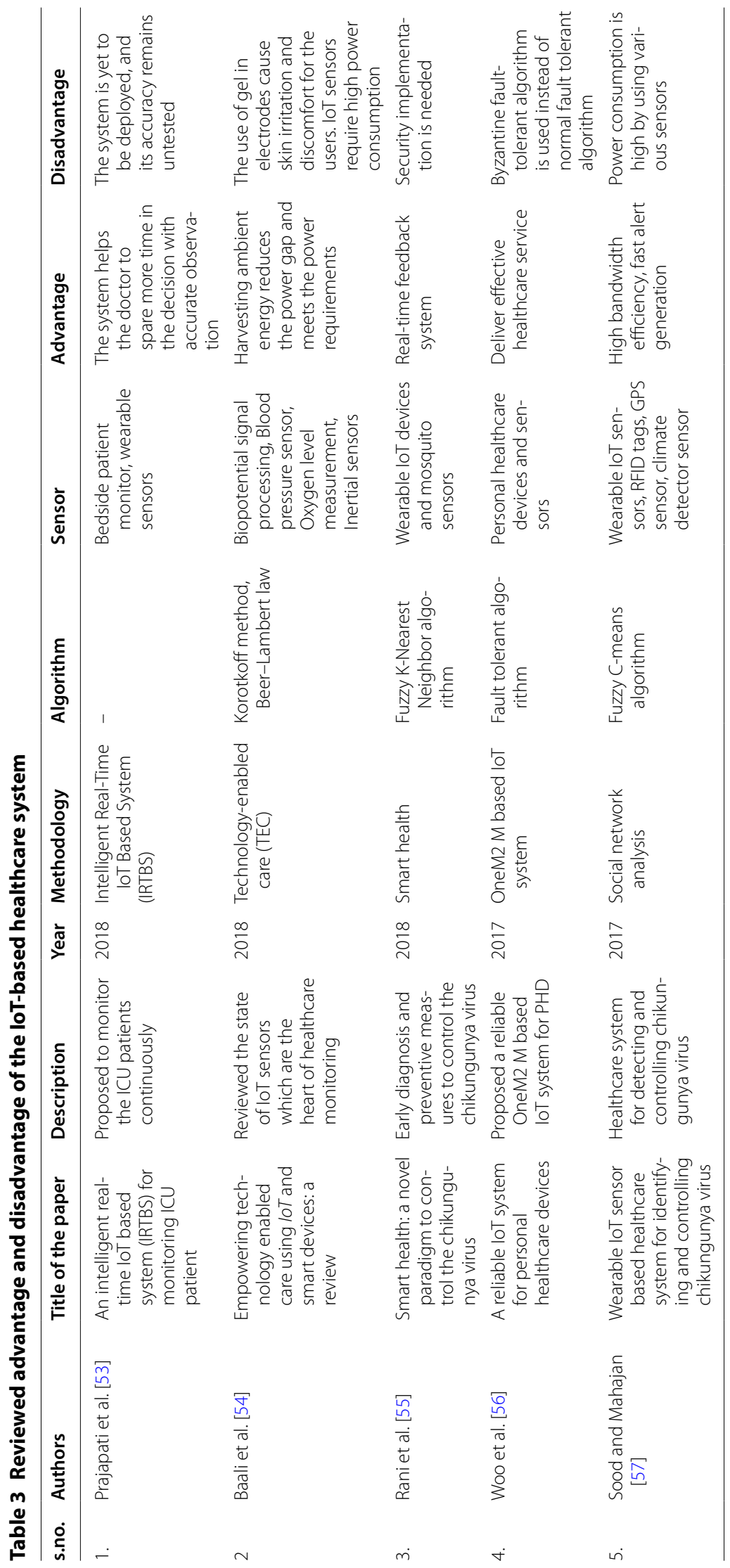


smart cities to monitor the patients real-time. Rani et al. [55] implemented the concept of smart health with the advancement of the IoT. The wearable devices and IoT sensors used to monitor the user's activities, and the data are processed at the edge of the network to reduce the cost and latency time. In traditional health system patients visit doctors directly for the illness and routine checkup. In E-health the patients and doctors communicate with the help of information and communication technologies. In the Telehealth system, the patients are monitored in the remote care through telecommunication technology. The smart health is the extension of E-health and Telehealth, where real-time sensors monitor the patients, diagnose and alert message sent to doctors immediately with efficient decision making, fast communication, and fast delivery of data. The authors designed the architecture of smart health in which patients register through mobile phones and get a unique id number. Using their id number, they update their symptoms frequently. Mosquito sensors are used to find the breeding sites and map that into google for uninfected users, the government via GPS.

In the personal healthcare system, sensors are denoted as Application DedicatedNode-Application Entity (ADN$\mathrm{AE})$ that monitor and gathered the surrounding data and communicated to the Middle Node-Common Service Entity (MN-CSE). Woo et al. [56] proposed a reliable oneM2 M based IoT system for real-time monitoring Personal Healthcare Devices (PHD). MN-CSE is the efficient communicator between AND-AE and Infrastructure Node-Common Service Entity (IN-CSE). The OneM2 M protocol is used for communication and doctors can access the PHD biomedical data. The workflow of the system is depicted as a communication message flow between PHD and the gateway. System failures occur due to power shortage, hardware malfunction, software bugs, or environmental hazards. The fault-tolerant algorithm is used to recover the data from system failure. Here, faulttolerance purposed with daisy chain where all gateways are connected in a chain to store backup copies of previous gateway ADNs. At the same time, two gateways (server) fault can be recovered.

In developing countries, diagnosis solution is not easily affordable and accessible. Sood and Mahajan [57] designed a system architecture consists of three layers: a wearable IoT sensor layer, the fog layer, and the cloud layer. Wearable IoT sensor layer collects data from the location sensor, health sensor, meteorological sensor, environment sensor and drug sensor. The health data, location data, meteorological data, environment data, and medicinal data are collected and moved to the fog layer. Fog is the intermediate layer between IoT sensors and cloud to process and analyze all the tracked data in real-time. The data further preprocessed and stored in the cloud layer. The fuzzy C-means algorithm is used to classify the users into infected and uninfected based on their symptoms. In the fog layer, once the user is diagnosed, then an alert message is sent to the users mobile whether they are infected or not. If they are infected, the sensors monitor them frequently and alert message pass to doctors and hospitals. In the cloud layer, social network analysis [58-60] (SNA) graph is generated for the infected users and mosquito breeding sites. The system uses information fragmentation and key sharing mechanism to avoid unlicensed access to data and provide security. The system experiments with different classification algorithms like Naïve Bayes, fuzzy C-means, fuzzy K-nearest neighbor, and neural network. The fuzzy $\mathrm{C}$-means produce high accuracy compared to other classification algorithms.

\section{Development of healthcare sector in the Big Data era}

Big Data analytics [61] in healthcare have been used to predict diseases, cure epidemic diseases, avoid preventable deaths and improves the quality of life. The role of Big Data architecture in healthcare is depicted in Fig. 4. The system collects the wearable IoT sensor data, climatic data, temperature data, environmental data, location data and medical data in different forms such as structured, unstructured, and semi-structured data. Then the data are integrated and visualized in cloud computing. The data are pre-processing in-database analytics by extracting, cleaning, statistical analysis, and the decisions are forward to doctors, remote users, and ambulance. Table 4 represents the merits and demerits of the Big Data in the healthcare system.

Wearable sensor devices continuously monitor the users and gather a large amount of structured and unstructured data called Big Data. From huge data, useful information is segregated for decision making is a tough process. Cloud computing used to store and manage the data on the remote server hosted on the internet cause more latency. To reduce the latency and improve the efficiency fog layer is introduced before the cloud to gather, analyze and process the data. Finally, the data are stored in the cloud to take a fast decision in real-time. IoT devices connected to a network to collect and exchange large data, i.e., Big Data. It is difficult to store, manage, analyze the meaningful information in the Big Data related to healthcare. Manogaran et al. [62] proposed a new architecture for IoT devices to store and process the sensor data (Big Data) for healthcare applications. The authors designed the Meta Fog-Redirection (MF-R) with Grouping and choosing (GC) architecture for IoT and Big Data ecosystem and to protect and prevent the Big 


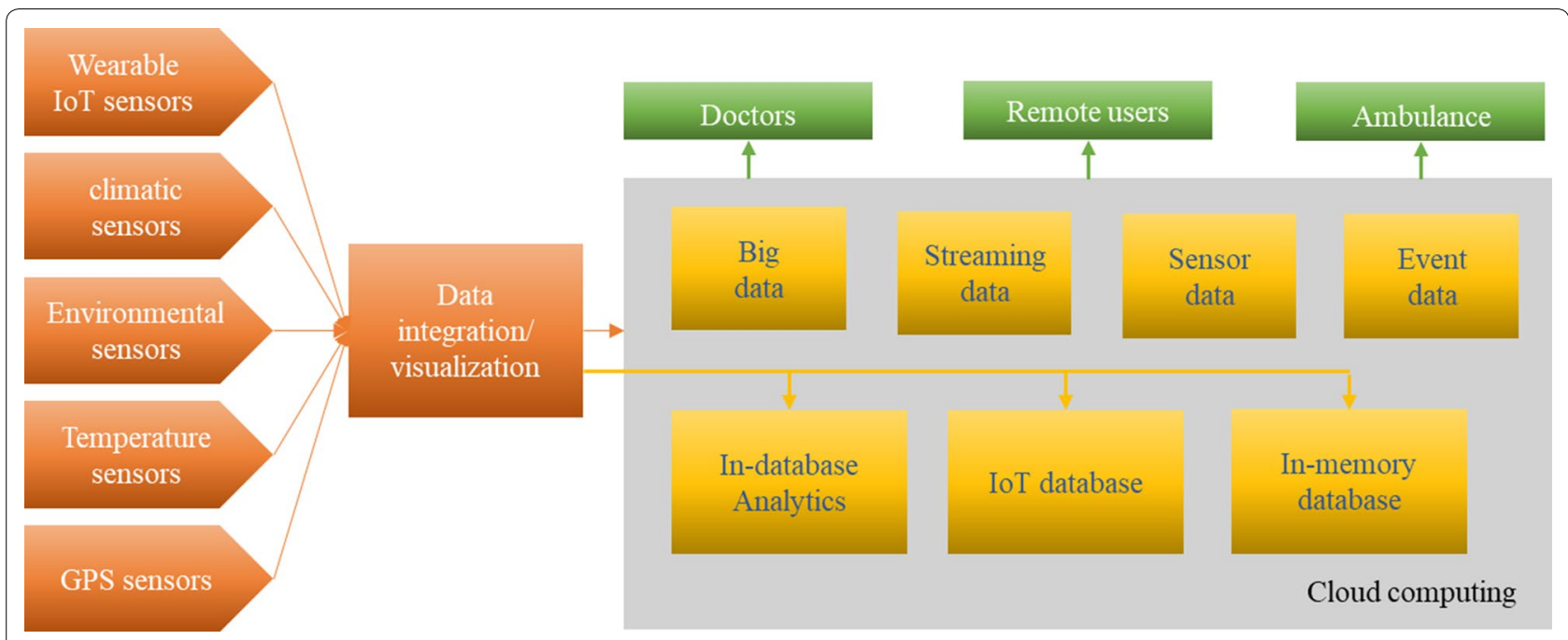

Fig. 4 Big Data architecture related to healthcare

Data from trespassers. IoT devices collect the user's heart rate, respiratory rate, body temperature, blood pressure, sugar rate through sensors and move to fog for further processing. During an emergency, the alert message sent to doctors through fog computing. In the cloud platform, Apache Pig and Apache HBase are used to store the Big Data.

For real-time remote monitoring, handling Big Data is the important challenge in healthcare decision making. Patients prioritization in telemedicine is an issue in Big Data analytics. Kalid et al. [63] connect the patient prioritization and real-time remote monitoring of healthcare services through Big Data six 'Vs.'Telemedicine for chronic diseases (heart disease, blood pressure, diabetic) needs continuous remote monitoring of patients through sensors. Sensors gather user's information in real-time and telemedicine provide healthcare services via doctors. Increasing patients leads to the increase in the problem of scalability, i.e., Priority overhead whom to serve first. To provide sufficient telemedicine services, they check the population aging and disaster into account. Patients prioritization occurs in three areas: surgery, transplantation, and surgical room. To solve, triage is used to decide the order of patients based on the severity.

In the emerging world, the IoT and Big Data analytics play a vital role in the smart healthcare system. Firouzi et al. [64] reviewed the advanced healthcare system with the application of wearable devices and sensors and the need for Big Data to inform these devices. The healthcare IoT system had three components: (i) body sensor area network used to record the patient's behavior data and environmental information such as temperature, humidity, date, time via body-wear sensors or implanted sensors. (ii) Internet-connected smart gateway, i.e., fog layer or local access network performs data preprocessing, protocol conversion, data filtering and mining and local notification to the patients. (iii) Cloud and Big Data support layer to store the all sensor information, analyzed, predict the decision and generate a message to the caregivers in need of an emergency.

Electronic Health Records (EHR) have the volatile growth of medical and health data need to a massive analysis of data based on Big Data analytics. First, the hospital dataset for outpatients are collected and more doctors are needed to treat them. Hu et al. [65] designed the Simultaneously Aided Diagnostic Model (SADM) to increase the working efficiency of outpatient doctors and decrease their working strength. The functions of the designed model are data acquisition, data storage, data preprocessing, feature extraction, machine learning, performance testing and finally instantaneous aided diagnosis to reference indices for the doctors. Data acquisition gathers outpatients' medication records, treatment schemes, treatment expenses, treatment process results, inspection results and image information. Machine learning trains the data with past medical dataset using Support Vector Machine and Neural Network algorithm to classify the hyperlipidemia.

In the today's emerging medical world, Big Data analytics role is to process a large volume and variety of data and store it as an efficient way to extract, update and delete the data easily. The data are stored in a multidimensional matrix called tensor based on concept hierarchy of attributes in the abstraction level. Sandhu et al. [66] proposed a tensor- and $\mathrm{GrC}$ - based data mining approach to address the three challenges like abstraction, 


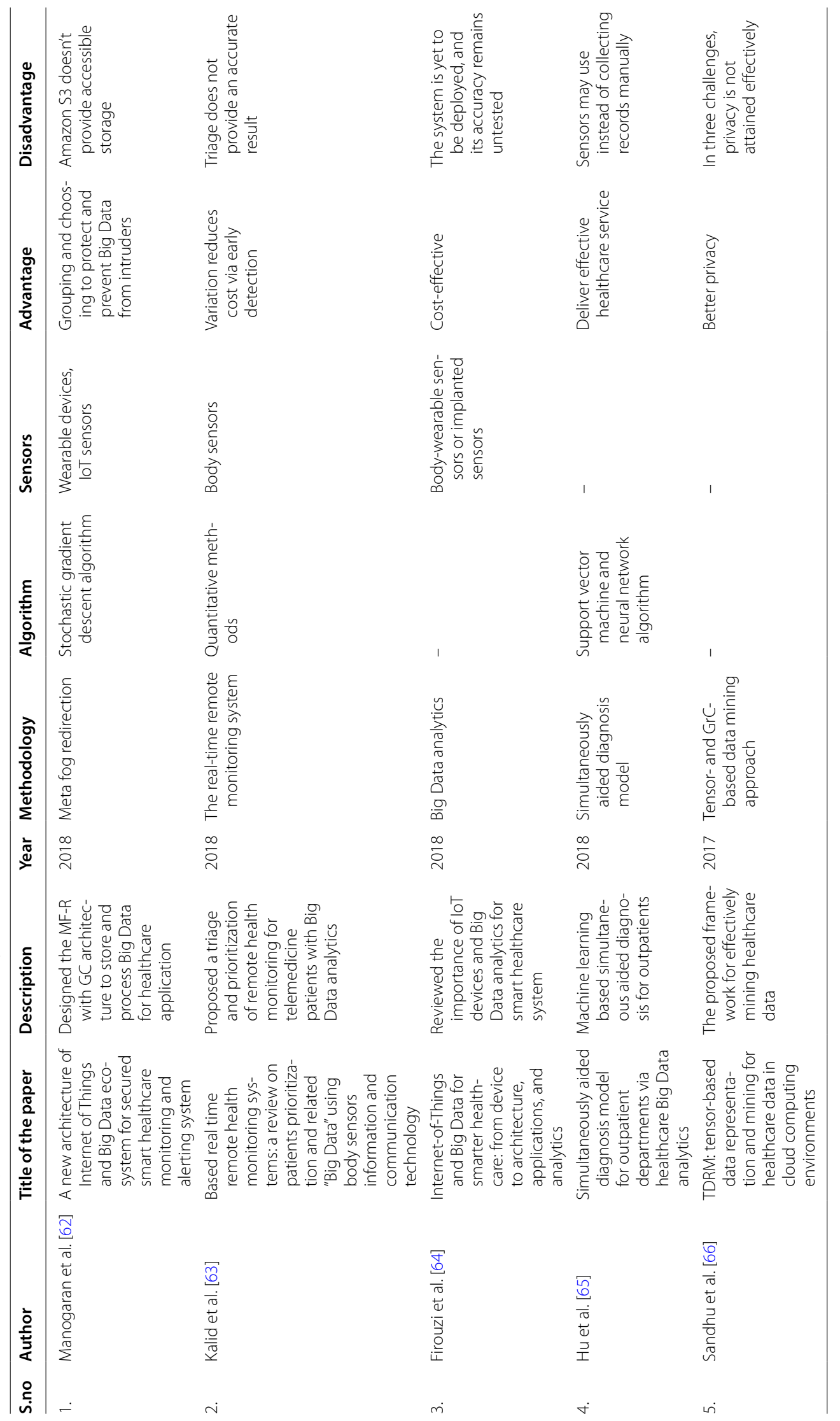


heterogeneity, and privacy. Granularity Computing ( $\mathrm{GrC})$ is an art of solving computing problems at the different level of granularity and extracting relevant information by removing irrelevant details from the available dataset. The proposed framework divided into three phases for effectively mining healthcare data. Data matrix is the first phase in which raw text data, medical data, audio, videos are stored in Health data Tensor (HdTr). In the second phase, using concept hierarchy, the data matrix is spanned over the different level of granularity. The third phase, the granules are used for query processing and final result formation. The performance evaluation is tested by experimenting with tensor-based data representation results in fast computation and lower cost compared to PARAFAC2 and CANDELINC. The diabetic dataset is converted to tensor format using tensor toolbox available in MATLAB [67] for the proposed application.

\section{Interactive healthcare applications with smartphones}

The smartphone is the most popular communicating device grown rapidly and emerge as a convenient tool in healthcare application in terms of sensing, smartness, and connectivity. Interactive healthcare application using a smartphone is depicted in Fig. 5. The smartphone with sensors tracked the patient's body temperature, blood pressure, skin temperature, accelerometer, moisture, climatic factors, etc., with the help of Bluetooth/WiFi, 3G/4G network. The information is stored in cloud computing and delivered to doctor's and family members mobile phones through SMS or emails with the help of network connectivity. The advantages of using mobile in healthcare application are real-time communication, increasing diagnosis accuracy by monitoring continuously, seamless flow of patients' information, and direct patients' record management. As shown below, Table 5 depicts the interactive usage of the smartphone in a healthcare application.

In both developed and developing countries, the number of greying population is increased with chronic diseases like diabetes, hypertension, heart disease, cholesterol, etc., and need continuous monitoring to diagnose at an early stage. Leu et al. [68] proposed the smartphone-based real-time monitoring of physiological data through Wireless body sensors. Sensors 24/7 continuously monitor the patients connected to the smartphones by which users carry wherever they go and always close to the sensor. The authors designed a Mobile Physiological Sensor System (MoPSS) which collects users physiological information like blood pressure, ECG, heart rate, temperature, blood glucose and oxygen saturation data from the smart shirt wearable by the user. The smart shirt allows us to collect the real-time physiological data which is then uploaded to the health cloud after displaying on a smartphone. The smart shirt wearable patients feel comfortable and move easily. The system provides real-time feedback and alert be generated directly, so the caregivers take care of the patients at the early stage. Real-time transmission of data is possible with the help of wireless protocols like Bluetooth, WiFi, ZigBee, GPRS, and 3G/4G. The Medium Access Protocol

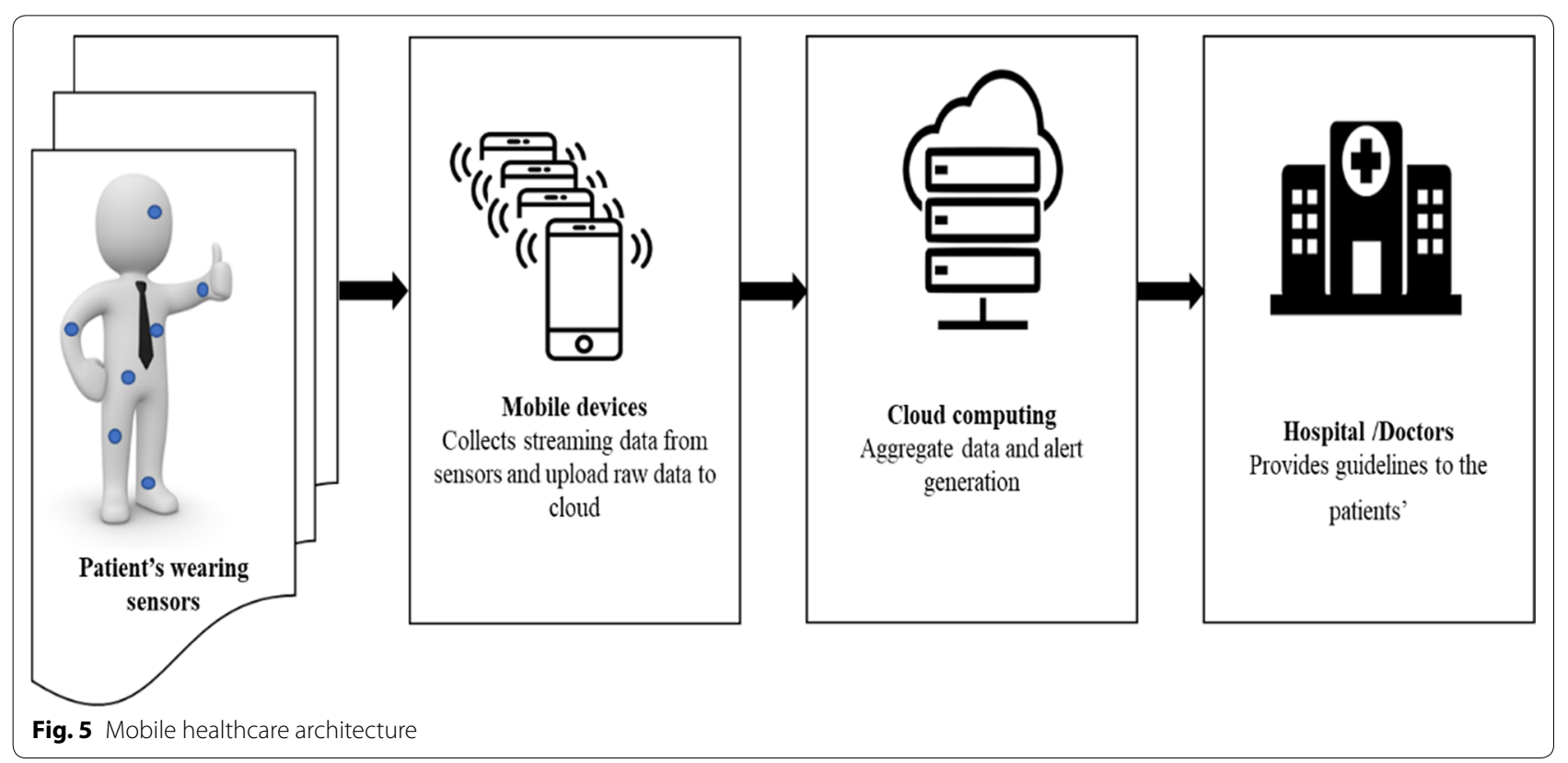




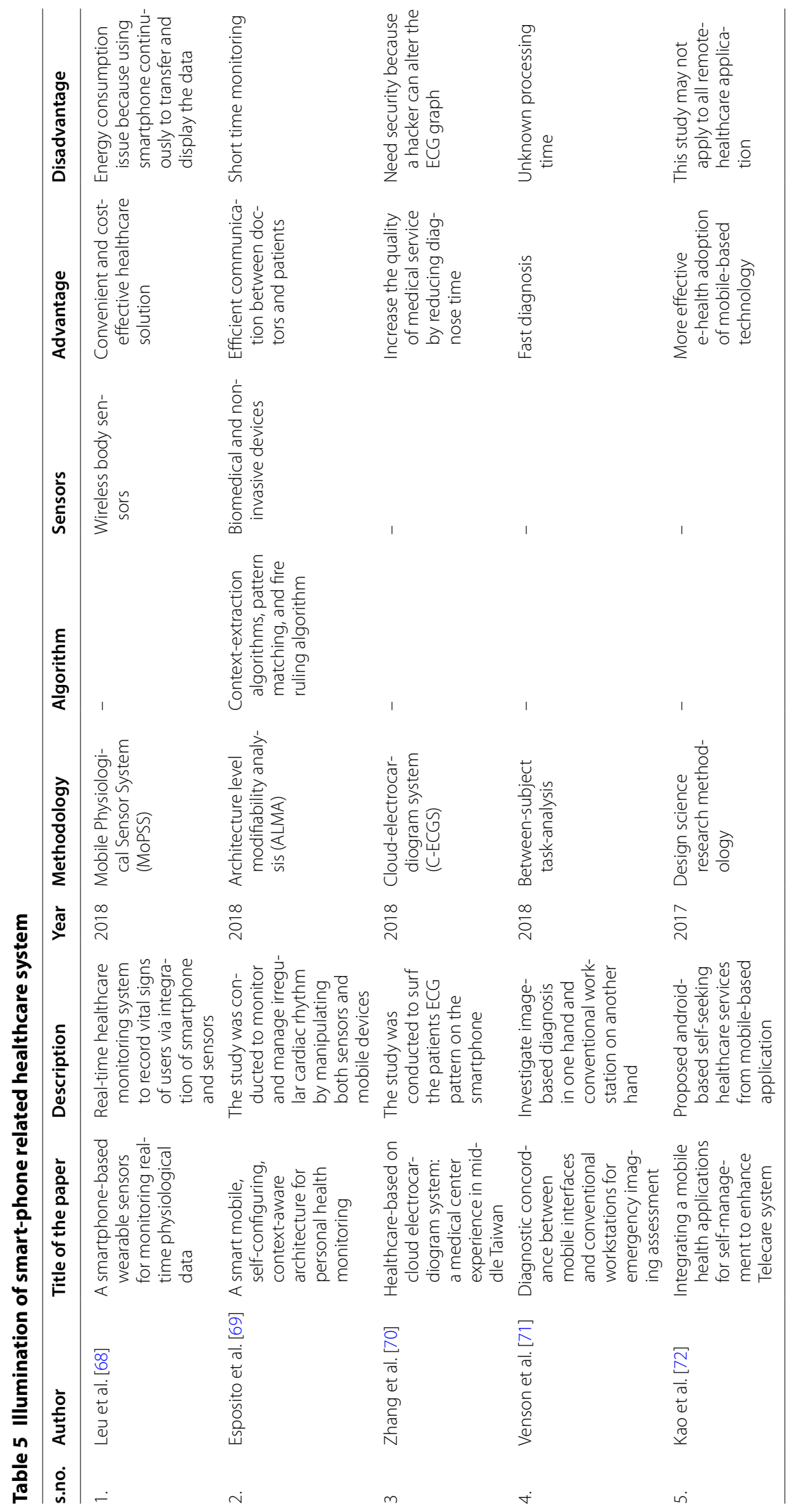


(MAC) protocol is used to reduce the power consumption, latency and packet loss rate.

The new emerging trend of smartphone healthcare application along with cellular communication networks and wireless sensors is a tremendous improvement in technology. Esposito et al. [69] proposed an ontologybased model for personal health monitoring. Here, the biomedical and non-invasive sensors attached to the patients collected periodic information about their movements, vital signs and sent to mobile phones. The information is stored and verified with personalized medical rules already deployed in mobile phones. The information is moved to caregivers frequently, and severity information is conveyed to the doctors through SMS or email. The authors presented four-tier architectural layers in which low layer information only known by next layer: (i) Sensing tier collects biomedical information and store it in mobile phones. (ii) Perceptual tier extracts vital signs and contextual information. (iii) Reasoning tier detects possible anomalies by incorporating specific rules. (iv) Actuating tier communicates with doctors regarding emergency by sending email or text messages. High-level ontology and low-level ontology for data shared with the components is described, and the system is implemented in Java and released to run on Android Operating System (AOS). The proposed architecture has been employed to monitor cardiac arrhythmias by Amiigo bracelet in a real-time application. However, the model needs improvement in the continuous monitoring because the life of the battery reduced fastly as per the usage of the smartphone.

Electrocardiogram (ECG) plays a vital role to measure the electrical activities of the heartbeat over a period by placing electrodes on the skin surface. It is the best method to diagnose the blockage of blood flow in the heart muscle, i.e., Acute Myocardial Infraction patients. Traditionally it takes more time to admit the patients in the emergency ward and to diagnose the heart attack. Later, $\mathrm{PC}$ is connected to the intranet of the hospital to verify the ECG. In PC, doctors have to download and install the Picture Archiving and Communication System (PACS) image browser to check the patients ECG. All those methods take additional time to diagnose and result in the increase of mortality rate. Zhang et al. [70] proposed a cloud-based Electrocardiogram system attached to a smartphone to browse the patients ECG pattern on the mobile screen. Cloud computing and mobile environment jointly emerge and paid a way to the healthcare system in early detection. The authors designed the architecture describe the workflow of cloud-based electrocardiogram system in which comprises six subsystems. In medical operation subsystem, the ECG report is sent to ECG server in the form of a graph. The main system had the details of patients such as name, age, gender, reference number, medical record, shift table of cardiologists, and ECG graph to C-ECGS. The SMS deliver the ECG graph to the consultant cardiologist for further reference during an emergency. As a result, mobile saves the treasured time, reduces mortality and allows doctors to diagnose remotely.

The image-based diagnosis process like MRI and CI need to interpret image interact with the dedicated workstations. Each workstation had highly trained technical users like medical physicist and radiologist to examine the images connected with the picture archiving and communication system. The results may be random and take a long time to diagnose. To overcome this problem, Venson et al. [71] proposed an image assessment through mobile devices with speed, complexity, and power to examine the real-time interpretation of radiology exams. A smartphone recently had a huge variety of radiologyrelated application for image interpretation. The authors designed the experiment to study the diagnostic concordance between mobile and workstations to diagnose the image. In most cases, mobile diagnose results are same as the full-featured workstation. However, lack of some tools in the mobile viewer to diagnose image-based assessment.

Chronic illness like diabetes, heart diseases, strokes, asthma, are growing rapidly needs the availability of healthcare resources. Kao et al. [72] developed an Android-based self-management application for homebased healthcare services. The application described the telecare technology which allows patients to consult with doctors as an online service. The system examined with various methodologies like telecare service model development, design science perspective, system architecture and software development, requirement modeling and implementation. The telecare architecture connects the home box with blood pressure gauge and Android OS smartphone to monitor the vital sign and inform clinical staffs and remote patients.

\section{Current challenges in the personalized healthcare}

In the healthcare applications, data privacy and security are the main issues as it transfers over the unsecured channel. The patient's medical data consists of personal information, health conditions, diagnostic reports, and its related treatments are sensitive. Hackers can modify the health-related information which results in misdiagnosis, or inaccurate assessment of diseases leads to inappropriate treatment and thus increases mortality rate. The transmission of medical information monitored through IoT devices is susceptible to security issues. Data security is considered as an essential factor for healthcare applications as it transmits user's sensitive information. 
At the hardware level, the security issues are found in terms of compatibility due to the requirements of each component such as the operating system, storage space and network architecture. At the software level, the security issue arises due to the nature of software used, as some of the software supports all programming language while other supports only specific software. For secure communication between IoT devices, SSL/TLS protocol is widely used. Cryptographic techniques can be used to provide secure communication and require more energy and processing power which are not feasible for wireless sensors and computing devices used in healthcare applications. Developing algorithms and schemes to protect the health-related information from unauthorized users is a challenging issue. Schematic errors in the stage of design and implementation also result in security risks.

Healthcare system faces many challenges due to senior population and increase of chronic diseases in various countries. In the healthcare system, another issue is a shortage of nursing facility and high QoS with low cost. Today we are wasting more time in hospitals by physically measuring biometric status and transfer the data between systems to store and access later. Manual entry of data have bit error, and difficult to access various types of data like records, image data. IoT devices and biosensors used in healthcare applications monitor blood pressure, body temperature, heart rate, glucose level continuously. By wearing sensors uninterruptedly may cause skin rashes and also consumes more power. For example, continuously nursing Ambulatory BP monitor the patients over $24 / 7$ at regular interval might cause bruises beneath the cuff, disturbance in their sleep and painful at high BP. Another challenge is a data processing at sensors due to resource constraints like the high cost of energy. Biopotential signal processing such as ECG, EEG, monitoring through sensors reduces the human error still need a low power consumption user-friendly sensor devices.

Big Data carries an overflow of real-world healthrelated data in a structured and unstructured form for monitoring patients 24/7. The evolution of technologies shifted the traditional mode of accessing data to six V's. Storage challenge is postured by volume, velocity, and variety of Big Data analytics. The traditional storage system is difficult to achieve in Big Data analytics; so cloud storage system like Amazon S2, Amazon EC2, Elastic Block Store provides a solution to the Big Data analytics by offers unlimited storage system with fault tolerance. Along with storage, another challenge is transferring Big Data to cloud service with reduced cost and increased speed. Here; the main issues are storage, retrieval of information and additionally extracting meaningful patterns using various algorithms. In the cloud, most works rely on Amazon Elastic Compute Cloud (Amazon EC2) to provide resizable compute capacity in the cloud. The cloud platform is not utilized efficiently to meet the rapidly growing needs for Big Data analytics and healthcare information. Alternative platforms like openCloud, owncloud, Dropbox, were deployed for effective experimental evaluation.

The major challenges while using smartphone-based healthcare application are inadequate to predict the diseases, low quality of data deliverance, performance analysis, data storage and privacy prevention. Utilizing smartphone supported self-monitoring sensors are costeffective and require a personalized treatment plan for a patient who is suffering from a specific illness. Though smartphone-based healthcare applications provide a great opportunity to improve the health, senior citizens cannot rely on smartphones all the time as they are not aware of technology and unable to use them effectively.

\section{Discussions on the promising solutions for the real-time problems}

IoT devices and sensors make users life more comfortable and provide a feasible solution to healthcare applications such as monitoring blood pressure, heart rate, diabetes, home care, vital sign monitoring like ECG, EEG. To visualize the usage of wearable devices in the healthcare system of older adults is a better understanding of the changes in their health conditions and promote their health management by themselves. Through wearable devices, the elders are eager to obtain the health advice to improve their living standards healthily [73]. The success of the healthcare system depends on security as well. Security in healthcare application using IoT devices, sensors, and actuators has been emerging research and worth for studying. In healthcare, patient's health data moved with the help of wireless network like Bluetooth, ZigBee, Wi-Fi, WiMAX, UMTS (Universal Mobile Telecommunication System), so malicious users must not be miscalculated. Since wireless network communication is not interned, malicious threats even pose important risks to the patients. In real-time healthcare applications, we want to provide strong security and privacy in the application design, deployment and implementation, i.e., include at the starting point of application. IoT sensor data related to healthcare requires authentication mechanism established at the server end to prevent improper access by the third-party user. Dynamic network topologies can adapt quickly to secure handover mechanism from one network to another network in the case of patients wearing sensors moves independently. To achieve high throughput for IoT health monitoring and efficient sensing, both communication cost and computation should be cost-effective. Healthcare monitoring application calculates synchronization time for 
end-to-end delay of packets. In real-time analysis, time synchronization delay between sensors and base station can be eliminated with the help of 4G technologies in the developing countries. Time synchronization is important in case of tracking the location of the patients who wear sensors for data collection, data aggregation, data routing, sensing and patient tracking. Healthcare application using sensors and mobile discover security as a special issues needs:(a)Elliptic curve cryptography: Current studies exposed that IoT sensors and devices may be practical with public key cryptography compared to the private key cryptography in terms of high cost and time complexity. Healthcare application with patients' information should be authenticated with public keys will be highly required. (b) Homomorphism: Homomorphic encryption [74] will make sensitive data transfer between users and cloud computing perfectly secure. Fully homomorphic encryption assists arbitrary computation with any Boolean gate allow to compute every computation (addition and multiplication) on the encrypted data. Partial Homomorphic Encryption allows certain computing computation (addition or multiplication) on the encrypted data. To resolve streaming patients' healthcare information and to provide security, homomorphic encryption will address in a better way. (c)Secret key cryptography: Also called symmetric key cryptography which is superior and easy to implement compared to public key cryptography regarding cost and time complexity. However, the problem in secret key cryptography occurs fault in the distribution of keys, i.e., a copy of the secret key should be shared at each end without any loss. Therefore, healthcare application needs to design a flexible and efficient key distribution protocol. (d)Secure Data Delivery: Special attention is essential for sensor network to route the data to the patient's mobility in multi-way without a loss for wireless healthcare applications. (e) Security and Quality-of-Service (QoS): Using IoT devices and sensor, not only security is the main concern. Jointly provide QoS with security to the users become healthcare application effective and more supportive of the people. (f) On-demand authentication: Healthcare application require specific authentication for the ubiquitous healthcare system gathers physiological, personal and environmental information by context-aware approaches for authenticating a particular patient.

Examining well-organized ways to utilize mobileBig Data come with better services in the IoT domain especially in smart healthcare services. Mobile-Big Data analytics was explored with a distributed framework on various parallel spark workers with the iteration of MapReduce (Hadoop) task. Integrating contextual information [75] along with IoT sensor data help to understand the environment's situation for quick data analytics and reasoning in a healthcare application. We cannot characterize the patient's health only with the help of physiological status. For example, Blood pressure of a human is measured using various IoT devices and sensors. By using contextual information like whether the person is running or idle can be integrated along with this sensor to get the exact seriousness of the situation. If the contextual information says that the person is running, then it is natural that the person will have high blood pressure, and there is no need to take any action. At the same time if the contextual information says that the person is idle and still the person has high blood pressure, then there is a need for an action to be taken immediately. The deployment of IoT Big Data analytics of healthcare application requires new protocols for online provisioning of cloud or fog on the new steam data.

\section{Conclusions}

Healthcare system has developed with the advance of smart IoT devices and sensors, cloud computing, fog computing and smartphones. Healthcare application developed for patient-centric prediction, prevention, diagnosis, and treatment. The wireless communication technologies have been advanced rapidly and available everywhere to handle the healthcare complications remotely. Healthcare ecosystem is evolved as an innovation in Big Data era with efficient decision making and support for a new lifestyle. Smartphone-based healthcare application can provide accurate and fast diagnostic prediction with controlling capability to address the challenges like high cost, storage and demands of professionals to attain directly. Wearable devices like smartwatches, smartphones, smart shirt, smart bracelets, smart clips, headbands, and smart clothing detect user's heart rate, body temperature, blood pressure and other activities. This survey investigates the systematic overview of the smart healthcare system and alert generation in the field of cloud computing, edge computing, Big Data analytics, IoT and mobile based applications with unique architectures. Exploring the pros and cons of each paper in the survey with different methodology and algorithm used. Healthcare applications and difficulties in real-time tracking using IoT devices have been discussed in detail. A security protocol for IoT healthcare monitoring devices needs further improvement by providing secure communication between devices and server, for protecting the individual privacy as well as to prevent data misuse or hack by an unauthorized entity. Monitoring patient health in real time is boon for medical field as a whole but to make the whole set-up work requires a robust telecommunication network and efficient use of 
limited wavelength available. Nowadays all sensing data will deliver users health data on their mobile phones to monitor their health behavior and vital signs. Therefore, a health monitoring device that not only transfers data at a fast rate but also requires less load on the currently available infrastructure is the need of the hour. Diseases are diagnosed in their early stages by remotely checking patients health condition in real time. The exact and being accurate medicine, i.e., being precision will be the future of the healthcare system. As a future work, we intend to improve the functioning of the existing healthcare system securely by applying homomorphic encryption, as well as developing and assessing an algorithm of an enhanced scheme in a real-world healthcare environment. We also plan to study the possibilities of homomorphism security mechanism to build secure healthcare application for the upcoming future generation.

\section{Author details}

${ }^{1}$ School of Computing, SASTRA Deemed University, Thanjavur, India. ${ }^{2}$ School of Computing Science and Engineering, Vellore Institute of Technology, Chennai, India.

\section{Acknowledgements}

The authors are grateful to Science and Engineering Research Board (SERB), Department of Science \& Technology, New Delhi, for the financial support (No. YSS/2014/000718/ES). Authors express their gratitude to SASTRA Deemed University, Thanjavur, for providing the infrastructural facilities to carry out this research work.

Received: 29 May 2018 Accepted: 12 August 2018

Published online: 20 September 2018

\section{References}

1. Ali Z, Hossain MS, Muhammad G, Sangaiah AK. An intelligent healthcare system for detection and classification to discriminate vocal fold disorders. Future Gen Comput Syst. 2018;85:19-28.

2. Saxena SK, Elahi A, Gadugu S, Prasad AK. Zika virus outbreak: an overview of the experimental therapeutics and treatment. VirusDisease. 2016;27(2):111-5.

3. Yang $\mathrm{G}$, et al. A health-loT platform based on the integration of intelligent packaging, unobtrusive bio-sensor, and intelligent medicine box. IEEE Trans Ind Inform. 2014;10(4):2180-91.

4. Yan Y, Li Q, Li H, Zhang X, Wang L. Open Access: A home-based health information acquisition system. Health Inform Sci Syst. 2013;1:12.

5. Khan M, Han K, Karthik S. Designing smart control systems based on Internet of Things and Big Data analytics. Wirel Pers Commun. 2018;99(4):1683-97.

6. Nachankar PJ, Somani MG, Singh DM, Katkar SN. IOT in agriculture. Int Res J Eng Technol. 2018;5(4):2395.

7. Paulraj GJL, Francis SAJ, Peter JD, Jebadurai IJ. Resource-aware virtual machine migration in loT cloud. Future Gen Comput Syst. 2018:85:173-83

8. Bhatia M, Sood SK. Exploring temporal analytics in fog-cloud architecture for Smart Office HealthCare. Mob Netw Appl. 2018. https://doi. org/10.1007/s11036-018-0991-5.

9. Wu T, Wu F, Redoute J-M, Yuce MR. An autonomous wireless body area network implementation towards loT connected healthcare applications. IEEE Access. 2017:5:11413-22.

10. Jalal L, Popescu V, Murroni M. IoT architecture for multisensorial media. In: 2017 IEEE URUCON, 2017, pp. 1-4.
11. Kawthankar S, Raut C. A survey on smart automobiles using Internet of Things for digital India. Int J Recent Trends Eng Res. 2017;3(5):27-33.

12. Gowda M et al. Bringing loT to sports analytics. In: 14th \{USENIX\} symposium networked system design implementation, \{NSDI\} 2017, Boston, MA, March 27-29, 2017, pp. 499-513.

13. Kim Y, Park Y, Choi J. A study on the adoption of IoT smart home service: using value-based adoption model. Total Qual Manag Bus Excell. 2017;28(9-10):1149-65.

14. Jo H, Yoon Yl. Intelligent smart home energy efficiency model using artificial TensorFlow engine. Hum Centric Comput Inf Sci. 2018. https:// doi.org/10.1186/s13673-018-0132-y.

15. Venkatesh K, Reddy PR. IOT based home automation using Android. Int J Mag Eng Technol Manag Res. 2017;3(4):317-20.

16. Jin Y, Ji S. Mapping hotspots and emerging trends of business model innovation under networking in Internet of Things. EURASIP J Wirel Commun Netw. 2018;2018(1):96.

17. Peng $H$, Tian $Y, L i L$, Yang $Y$, Wang D. Secure and energy-efficient data transmission system based on chaotic compressive sensing in body-tobody networks. IEEE Trans Biomed Circuits Syst. 2017;11(3):1-16.

18. Wang Y, Kung L, Wang WYC, Cegielski CG. An integrated Big Data analytics-enabled transformation model: application to health care. Inf Manag. 2018;55(1):64-79.

19. Wang Y, Kung L, Byrd TA. Big data analytics: understanding its capabilities and potential benefits for healthcare organizations. Technol Forecast Soc Change. 2018;126:3-13.

20. Oppitz M, Tomsu P. Inventing the cloud century. Cham: Springer; 2018.

21. Yang $C$, Yu M, Hu F, Jiang Y, Li Y. Utilizing cloud computing to address big geospatial data challenges. Comput Environ Urban Syst. 2017;61:120-8.

22. Yang C, Huang Q, Li Z, Liu K, Hu F. Big Data and cloud computing: innovation opportunities and challenges. Int J Digit Earth. 2017;10(1):13-53.

23. Upadhyaya J. Higher education: a critical survey and innovative model, 2017, pp. 137-140.

24. Stergiou C, Psannis KE, Kim BG, Gupta B. Secure integration of loT and cloud computing. Future Gen Comput Syst. 2018;78:964-75.

25. Kraemer FA, Braten AE, Tamkittikhun N, Palma D. Fog computing in healthcare-a review and discussion. IEEE Access. 2017;5(2169):9206-22.

26. Choo K-KR, Lu R, Chen L, Yi X. A foggy research future: advances and future opportunities in fog computing research. Future Gen Comput Syst. 2018;78:677-9.

27. Roca D, Milito R, Nemirovsky M, Valero M. Fog computing in the Internet of Things. Cham: Springer; 2018.

28. Liu X, Deng RH, Yang Y, Tran HN, Zhong S. Hybrid privacy-preserving clinical decision support system in fog-cloud computing. Future Gen Comput Syst. 2018;78:825-37.

29. Chen M, Yang J, Zhou J, Hao Y, Zhang J, Youn C. 5G-smart diabetes: toward personalized diabetes diagnosis with healthcare Big Data clouds. IEEE Commun Mag. 2018;56(4):16-23.

30. Xu D, Huang X, Guo J, Ma X. Automatic smartphone-based microfluidic biosensor system at the point of care. Biosens Bioelectron. 2018;110(March):78-88.

31. Harari GM, et al. An evaluation of students' interest in and compliance with self-tracking methods. Soc Psychol Personal Sci. 2017;8(5):479-92.

32. Pham M, Mengistu Y, Do H, Sheng W. Delivering home healthcare through a cloud-based smart home environment (CoSHE). Future Gen Comput Syst. 2018:81:129-40.

33. Nappi M, Ricciardi S, Tistarelli M. Context awareness in biometric systems and methods: state of the art and future scenarios. Image Vis Comput. 2018;76:27-37

34. Nakahara FA, Beder DM. A context-aware and self-adaptive offloading decision support model for mobile cloud computing system. J Ambient Intell Humaniz Comput. 2018. https://doi.org/10.1007/s1265 2-018-0790-7.

35. Verma P, Sood SK. Cloud-centric loT based disease diagnosis healthcare framework. J Parallel Distrib Comput. 2018;116:27-38.

36. Sareen S, Sood SK, Gupta SK. Secure Internet of Things-based cloud framework to control Zika virus outbreak. Int J Technol Assess Health Care. 2017;33(1):11-8.

37. Sandhu R, Gill HK, Sood SK. Smart monitoring and controlling of pandemic influenza A (H1N1) using social network analysis and cloud computing. J Comput Sci. 2016;12:11-22. 
38. Hossain MS, Muhammad G. Cloud-assisted Industrial Internet of Things (IloT) —enabled framework for health monitoring. Comput Netw. 2015;101:192-202.

39. Zogheib $\mathrm{E}$, et al. Prospective observational study on the association between serum mannose-binding lectin levels and severe outcome in critically ill patients with pandemic influenza type A (H1N1) infection. Lung. 2018;196(1):65-72.

40. Verma P, Sood SK. Fog assisted-loT enabled patient health monitoring in smart homes. IEEE Internet Things J. 2018;4662:1-8.

41. Sood SK, Mahajan I. A fog based healthcare framework for Chikungunya. IEEE Internet Things J. 2017;4662:1-8.

42. Negash B, et al. Fog computing in the Internet of Things. Cham: Springer; 2018.

43. Sareen S, Gupta SK, Sood SK. An intelligent and secure system for predicting and preventing Zika virus outbreak using Fog computing. Enterp Inf Syst. 2017;11(9):1-21.

44. Nandyala CS, Kim HK. From cloud to fog and loT-based real-time U-healthcare monitoring for smart homes and hospitals. Int J Smart Home. 2016;10(2):187-96.

45. Gia TN, Jiang M, Rahmani AM, Westerlund T, Liljeberg P, Tenhunen H. Fog computing in healthcare Internet of Things: a case study on ECG feature extraction. In: Proceedings of the 15th IEEE international conference computer information technology, CIT 2015, 14th IEEE international conference ubiquitous computer communication, IUCC 2015, 13th IEEE international conference dependable, automatic and secure, 2015, pp. 356-363.

46. Yu Q, et al. Automatic identifying of maternal ECG source when applying ICA in fetal ECG extraction. Biocybern Biomed Eng. 2018;38(3):448-55.

47. Narayan R, Rout JK, Jena SK. Progress in intelligent computing techniques: theory, practice, and applications, vol. 518. Singapore: Springer; 2018.

48. Lv Z, Zhang C, Zhou B, Gao X, Wu X. Design and implementation of an eye gesture perception system based on electrooculography. Expert Syst Appl. 2018;91:310-21.

49. Ferrari E, Cooper G, Reeves ND, Hodson-Tole EF. Surface electromyography can quantify temporal and spatial patterns of activation of intrinsic human foot muscles. J Electromyogr Kinesiol. 2018;39(February):149-55.

50. Fink $A$, et al. EEG alpha activity during imagining creative moves in soccer decision-making situations. Neuropsychologia. 2018;114(April):118-24.

51. Amin R, Kumar N, Biswas GP, lqbal R, Chang V. A light weight authentication protocol for loT-enabled devices in distributed cloud computing environment. Future Gen Comput Syst. 2018;78:1005-19.

52. Beuchat $P$, Hesse H, Domahidi A, Lygeros J. Optimization based selflocalization for loT wireless sensor networks. In: 4th IEEE World Forum on Internet of Things, pp. 712-717.

53. Prajapati B, Parikh S, Patel J. Information and communication technology for intelligent systems. In: ICTIS 2017, vol. 1, p. 3, 2018.

54. Baali $H$, Djelouat $H$, Amira A, Bensaali F. Empowering technology enabled care using loT and smart devices: a review. IEEE Sens J. 2017;18(5):1790-809.

55. Rani S, Ahmed SH, Shah SC. Smart health: a novel paradigm to control the chickungunya virus. IEEE Internet Things J. 2018;4662:1.

56. Woo MW, Lee JW, Park $\mathrm{KH}$. A reliable loT system for personal healthcare devices. Future Gen Comput Syst. 2018;78:626-40.

57. Sood SK, Mahajan I. Wearable IoT sensor based healthcare system for identifying and controlling chikungunya virus. Comput Ind. 2017:91:33-44.
58. Dutta S, Ghatak S, Dey R, Das AK, Ghosh S. Attribute selection for improving spam classification in online social networks: a rough set theorybased approach. Soc Netw Anal Min. 2018;8(1):7.

59. Marozzo F, Bessi A. Analyzing polarization of social media users and news sites during political campaigns. Soc Netw Anal Min. 2018;8(1):1.

60. Das K, Samanta S, Pal M. Study on centrality measures in social networks: a survey. Soc Netw Anal Min. 2018;8(1):13.

61. Raghupathi W, Raghupathi V. Big Data analytics in healthcare: promise and potential. Health Inf Sci Syst. 2014;2:3.

62. Manogaran G, Varatharajan R, Lopez D, Kumar PM, Sundarasekar R, Thota C. A new architecture of Internet of Things and Big Data ecosystem for secured smart healthcare monitoring and alerting system. Future Gen Comput Syst. 2018;82:375-87.

63. Kalid N, Zaidan AA, Zaidan BB, Salman OH, Hashim M, Muzammil H. Based real time remote health monitoring systems: a review on patients prioritization and related 'Big Data' using body sensors information and communication technology. J Med Syst. 2018;42:2.

64. Firouzi F, et al. Internet-of-Things and Big Data for smarter healthcare: from device to architecture, applications and analytics. Future Gen Comput Syst. 2018;78:583-6.

65. Hu Y, Duan K, Zhang Y, Hossain MS, Mizanur-Rahman SM, Alelaiwi A. Simultaneously aided diagnosis model for outpatient departments via healthcare Big Data analytics. Multimed Tools Appl. 2018;77(3):3729-43.

66. Sandhu R, Kaur N, Sood SK, Buyya R. TDRM: tensor-based data representation and mining for healthcare data in cloud computing environments. J Supercomput. 2017;74(2):592-614.

67. Saleh N, Kassem A, Haidar AM. Energy-efficient architecture for wireless sensor networks in healthcare applications. IEEE Access. 2018;6:6478-86.

68. Leu F, Ko C, You I, Choo KKR, Ho CL. A smartphone-based wearable sensors for monitoring real-time physiological data. Comput Electr Eng. 2018;65:376-92.

69. Esposito M, Minutolo A, Megna R, Forastiere M, Magliulo M, De Pietro G. A smart mobile, self-configuring, context-aware architecture for personal health monitoring. Eng Appl Artif Intell. 2018;67:136-56.

70. Zhang X-S, Leu F-Y, Yang C-W, Lai L-S. Healthcare-based on cloud electrocardiogram system: a medical center experience in middle Taiwan. J Med Syst. 2018;42(3):39.

71. Venson JE, Bevilacqua F, Berni J, Onuki F, Maciel A. Diagnostic concordance between mobile interfaces and conventional workstations for emergency imaging assessment. Int J Med Inform. 2018;113(2017):1-8.

72. Kao HY, Wei CW, Yu MC, Liang TY, Wu WH, Wu YJ. Integrating a mobile health applications for self-management to enhance Telecare system. Telemat Inform. 2017;12:011.

73. Zhou S, Ogihara A, Nishimura S, Jin Q. Analyzing the changes of health condition and social capital of elderly people using wearable devices. Health Inform Sci Syst. 2018. https://doi.org/10.1007/s13755-018-0044-2.

74. Xu J, Wei L, Zhang Y, Wang A, Zhou F, Gao C. Dynamic fully homomorphic encryption-based Merkle tree for lightweight streaming authenticated data structures. J Netw Comput Appl. 2018;107:113-24.

75. Rault T, Bouabdallah A, Challal Y, Marin F. A survey of energy-efficient context recognition systems using wearable sensors for healthcare applications. Pervasive Mob Comput. 2017;37:23-44.

Publisher's Note Springer Nature remains neutral with regard to jurisdictional claims in published maps and institutional affiliations. 